\title{
ASYMPTOTIC EFFICIENCY OF STATISTICAL ESTIMATES IN A COMPOUND POISSON MODEL
}

UDC 519.21

\author{
O. G. KUKUSH AND YU. S. MISHURA
}

\begin{abstract}
We consider maximum likelihood statistical estimates for the number of individuals in a biological population modelled by a compound Poisson process. We prove the local asymptotic normality and asymptotic efficiency of the estimates.
\end{abstract}

\section{INTRODUCTION}

One of the models used to construct statistical estimates for the number of individuals in a biological population was constructed by M. Thomas ([1]; see also [2]). This model can be specified by the following assumptions: the number of clusters (or parent species) has the Poisson distribution with parameter $\lambda_{p}>0$; any parent organism generates, independently of others, a random number of daughter species according to the Poisson distribution with parameter $\lambda_{D}>0$. Let $X$ denote the total number of individuals in a population. Then it has the Thomas distribution with parameters $\left(\lambda_{p}, \lambda_{D}\right)$, namely

$$
\begin{gathered}
\mathrm{P}\{X=k\}=e^{-\lambda_{p}} \sum_{r=1}^{k}\left(r \lambda_{D}\right)^{k-r}\left(\lambda_{p} e^{-\lambda_{D}}\right)^{r}(r !(k-r) !)^{-1}, \\
k=1,2, \ldots, \quad \mathrm{P}\{X=0\}=e^{-\lambda_{p}} .
\end{gathered}
$$

The problem to be solved by a statistician is to construct estimates for the parameters $\lambda_{p}$ and $\lambda_{D}$ and to study the behavior of the estimates. We consider a generalization of the Thomas model specified by the following assumptions: the number of parent species depends on time and is described by a homogeneous Poisson process $N(t)$ with parameter $\lambda_{p}>0$; any parent organism generates $\xi_{k} \geq 0$ daughter species; $N(t)$ and $\xi_{k}$, $k \geq 1$, are mutually independent; $\xi_{k}$ has the Poisson distribution with parameter $\lambda_{D}>0$. Then the total number of individuals in a population at a time $t$ equals

$$
X(t)=\sum_{k=0}^{N(t)}\left(1+\xi_{k}\right),
$$

which is a generalized point process with right continuous step trajectories that have the jumps

$$
\Delta X(t)=X(t)-X(t-0)=(\xi(N(t))+1) I\{\Delta N(t)=1\}, \quad \Delta X(t) \in \mathbf{N} .
$$

2000 Mathematics Subject Classification. Primary 62F10, 62F12, 60 G55.

Key words and phrases. Compound Poisson process, statistical estimates, asymptotic efficiency, local asymptotic normality.

The work is partially supported by INTAS grant No. 99-000-16. 
It is clear that $X(t)$ is a stochastically continuous homogeneous process with independent increments. Denote by $\nu(t, A)$ the number of those jumps of the process $X$ occurring up to a moment $t$ for which their heights belong to the set $A \subset \mathbf{N}$.

According to [3] $\S 11$, Theorems 1 and 2, and $\S 14$, Theorem 1], $\nu(t, A), t \geq 0$, is a stochastically continuous homogeneous nondecreasing process with independent increments having the Poisson distribution and such that $\mathrm{E} \nu(t, A)=\Pi(t, A)$, where $\Pi(t, \cdot)$ is a finite measure on the $\sigma$-field $2^{\mathbf{N}}$.

At first glance, the problem of estimation of parameters of the process $X(t)$ can be decomposed into two classical problems:

(a) to estimate the parameter $\lambda_{p}$ of the Poisson process $N(t)$ by the moments of jumps $t_{1}, t_{2}, \ldots, t_{k}, k=N(t)$, and

(b) to estimate the parameter $\lambda_{D}$ by the observations $\xi_{1}, \xi_{2}, \ldots, \xi_{k}$.

However, $k=N(t)$ is a random variable, and the problems (a) and (b) depend on each other. In such a situation the construction of efficient estimates for the parameters $\lambda_{p}$ and $\lambda_{D}$ becomes nontrivial.

\section{Maximum likelihood estimates of parameters $\lambda_{p}$ AND $\lambda_{D}$}

First we evaluate the measure $\Pi$.

Lemma 1. $\Pi(t,\{k\})=t \lambda_{p} \exp \left\{-\lambda_{D}\right\}\left(\lambda_{D}\right)^{k-1} /(k-1) !, k \geq 1$.

Proof. It is clear that

$$
\begin{aligned}
\mathrm{P}\{\nu(t,\{k\})=0\} & =\mathrm{P}\{N(t)=0\}+\sum_{r=1}^{\infty} \mathrm{P}\left\{N(t)=r, \xi_{1}+1 \neq k, \ldots, \xi_{r}+1 \neq k\right\} \\
& =e^{-\lambda_{p} t}+\sum_{r=1}^{\infty} \frac{\left(\lambda_{p} t\right)^{r}}{r !} e^{-\lambda_{p} t}\left(1-\frac{\left(\lambda_{D}\right)^{k-1}}{(k-1) !} e^{-\lambda_{D}}\right)^{r} \\
& =\sum_{r=0}^{\infty} \frac{\left[\left(\lambda_{p} t\right)\left(1-\frac{\left(\lambda_{D}\right)^{k-1}}{(k-1) !} e^{-\lambda_{D}}\right)\right]^{r}}{r !} e^{-\lambda_{p} t} \\
& =\exp \left\{-\lambda_{p} t \frac{\left(\lambda_{D}\right)^{k-1}}{(k-1) !} e^{-\lambda_{D}}\right\}, \quad k \geq 1 .
\end{aligned}
$$

Since

$$
\mathrm{P}\{\nu(t,\{k\})=0\}=\exp \{-\Pi(t,\{k\})\},
$$

the proof follows directly from (1).

Remark. Since $\nu(t,\{k\}), t \geq 0$, is a homogeneous Poisson stochastic process,

$$
\Pi(t,\{k\})=t \cdot \lambda_{\nu},
$$

where $\lambda_{\nu}=\lambda_{p} \frac{\left(\lambda_{D}\right)^{k-1}}{(k-1) !} e^{-\lambda_{D}}$ is the parameter of this process.

Now we consider the "unit" process $X_{1}(t)$ having the same structure as $X(t)$, but with parameters $\lambda_{p}=\lambda_{D}=1$. Denote by $P_{\lambda_{p}, \lambda_{D}}(t)$ and $P_{1,1}(t)$ the measures in the space of step functions $X(s), 0 \leq s \leq t$, and $X_{1}(s), 0 \leq s \leq t$, respectively. We evaluate the likelihood ratio of these measures and present it in the following result.

Lemma 2. The measures $P_{\lambda_{p}, \lambda_{D}}$ and $P_{1,1}$ are equivalent. Moreover,

$$
\frac{d P_{\lambda_{p}, \lambda_{D}}(t)}{d P_{1,1}(t)}(X(t))=\exp \left\{\sum_{k=1}^{\infty} \ln \left(\lambda_{p} e^{-\lambda_{D}}\left(\lambda_{D}\right)^{k-1}\right) \cdot \nu(t,\{k\})-t\left(\lambda_{p}-1\right)\right\} .
$$


Proof. The parameter of the Poisson process $\nu(t,\{k\}), t \geq 0$, with $\lambda_{p}=\lambda_{D}=1$ equals $\lambda_{\nu_{1}}=1 /(k-1)$ ! by the remark above. Therefore the measure $\Pi(t, A)=\sum_{k \in A} \Pi(t,\{k\})$ is equivalent to the measure $\Pi_{1}(t, A)$, and

$$
\rho(t, k):=\frac{d \Pi(t,\{k\})}{d \Pi_{1}(t,\{k\})}=\lambda_{p} \cdot e^{-\lambda_{D}}\left(\lambda_{D}\right)^{k-1} .
$$

By [3, $\S 46$, Theorem] and [4, Theorem 11] the measures $P_{\lambda_{p}, \lambda_{D}}$ and $P_{1,1}$ are equivalent, and their likelihood ratio equals

$$
\begin{aligned}
& \frac{d P_{\lambda_{p}, \lambda_{D}}(t)}{d P_{1,1}(t)}(X(t)) \\
& \quad=\exp \left\{\int_{0}^{t} \sum_{k=1}^{\infty} \ln \left(\lambda_{p} e^{-\lambda_{D}}\left(\lambda_{D}\right)^{k-1}\right) \cdot \nu(d s,\{k\})-\Pi(t,[1, \infty))+\Pi_{1}(t,[1, \infty))\right\} \\
& \quad=\exp \left\{\sum_{k=1}^{\infty} \ln \left(\lambda_{p} e^{-\lambda_{D}}\left(\lambda_{D}\right)^{k-1}\right) \cdot \nu(t,\{k\})-\lambda_{p} t+t\right\},
\end{aligned}
$$

whence the proof follows. Here we used the representation

$$
\Pi(t,[1, \infty))=\sum_{k=1}^{\infty} \lambda_{p} t e^{-\lambda_{D}} \frac{\left(\lambda_{D}\right)^{k-1}}{(k-1) !}=\lambda_{p} t .
$$

Now we are in a position to present the explicit formulas for the estimates.

Theorem 1. Maximum likelihood estimates of the parameters $\lambda_{p}$ and $\lambda_{D}$ constructed from observations $X(t)$ on the interval $[0, t]$ are equal to

$$
\widehat{\lambda}_{p}(t)=\frac{\nu(t,[1, \infty))}{t}, \quad \widehat{\lambda}_{D}(t)=\frac{\sum_{k=2}^{\infty}(k-1) \nu(t,\{k\})}{\nu(t,[1, \infty))} .
$$

Here we assume that $\frac{0}{0}=0$.

Proof. We transform the logarithm of the likelihood ratio obtained in Lemma 2:

$$
\begin{aligned}
& f\left(t, \lambda_{p}, \lambda_{D}\right)=\sum_{k=1}^{\infty} \ln \left(\lambda_{p} e^{-\lambda_{D}}\left(\lambda_{D}\right)^{k-1}\right) \cdot \nu(t,\{k\})-\lambda_{p} t+t \\
& \quad=\left(\ln \lambda_{p}\right) \nu(t,[1, \infty))-\lambda_{D} \nu(t,[1, \infty))-\lambda_{p} t+t+\left(\ln \lambda_{D}\right) \sum_{k=1}^{\infty}(k-1) \nu(t,\{k\}) .
\end{aligned}
$$

Differentiating the latter expression we obtain

$$
\begin{gathered}
f_{\lambda_{p}}^{\prime}=\frac{1}{\lambda_{p}} \nu(t,[1, \infty))-t, \\
f_{\lambda_{D}}^{\prime}=-\nu(t,[1, \infty))+\frac{1}{\lambda_{D}} \sum_{k=1}^{\infty}(k-1) \nu(t,\{k\}),
\end{gathered}
$$

whence the proof follows.

\section{Consistency and asymptotic normality of estimates}

Below we consider some properties of the estimates $\widehat{\lambda}_{p}(t)$ and $\widehat{\lambda}_{D}(t)$.

Lemma 3. The estimate $\widehat{\lambda}_{p}(t)$ is unbiased, $\mathrm{E} \widehat{\lambda}_{p}(t)=\lambda_{p}$. The estimate $\lambda_{D}$ is asymptotically unbiased, $\mathrm{E} \hat{\lambda}_{D}(t)=\lambda_{D}\left(1-e^{-\lambda_{p} t}\right)$. 
Proof. Put

$$
\begin{gathered}
\alpha_{k}=\alpha_{k}(t)=\frac{t \lambda_{p}\left(\lambda_{D}\right)^{q-1}}{(q-1) !} e^{-\lambda_{D}}, \\
\beta_{k}=\beta_{k}(t)=\sum_{q \in \mathbf{N} \backslash\{k\}} \frac{t \lambda_{p}\left(\lambda_{D}\right)^{q-1}}{(q-1) !} e^{-\lambda_{D}}, \\
\gamma=\gamma(t)=\alpha_{k}+\beta_{k}=\lambda_{p} t .
\end{gathered}
$$

Then $\mathrm{E} \widehat{\lambda}_{p}(t)=t^{-1} \Pi(t,[1, \infty))=\lambda_{p}, \mathrm{E} \widehat{\lambda}_{D}(t)=\sum_{k=2}^{\infty}(k-1) \mathrm{E} \frac{\nu(t,\{k\})}{\nu(t,[1, \infty))}$, and

$$
\begin{aligned}
\mathrm{E} \frac{\nu(t,\{k\})}{\nu(t,[1, \infty))} & =\sum_{l \geq 1, r \geq 0} \frac{l}{l+r} \mathrm{P}\{\nu(t,\{k\})=l\} \cdot \mathrm{P}\{\nu(t, \mathbf{N} \backslash\{k\})=r\} \\
& =e^{-\gamma} \sum_{l \geq 0, r \geq 0} \frac{1}{l+r+1} \cdot \frac{\alpha_{k}^{l}}{l !} \cdot \frac{\beta_{k}^{r}}{r !}=\alpha_{k} e^{-\gamma} \sum_{n=0}^{\infty} \sum_{l=0}^{n} \frac{1}{n+1} \cdot \frac{\alpha_{k}^{l} \beta_{k}^{n-l}}{l !(n-l) !} \\
& =\alpha_{k} e^{-\gamma} \sum_{n=0}^{\infty} \frac{1}{(n+1) !} \gamma^{n}=\frac{\alpha_{k} e^{-\gamma}}{\gamma}\left(e^{\gamma}-1\right)=\frac{\left(\lambda_{D}\right)^{k-1}}{(k-1) !} e^{-\lambda_{D}}\left(1-e^{-\lambda_{p} t}\right),
\end{aligned}
$$

whence

$$
\mathrm{E} \widehat{\lambda}_{D}(t)=\sum_{k=2}^{\infty}(k-1) \frac{\left(\lambda_{D}\right)^{k-1}}{(k-1) !} e^{-\lambda_{D}}\left(1-e^{-\lambda_{p} t}\right)=\lambda_{D}\left(1-e^{-\lambda_{p} t}\right) .
$$

The lemma is proved.

Theorem 2. The estimates $\widehat{\lambda}_{p}(t)$ and $\widehat{\lambda}_{D}(t)$ are strongly consistent: $\widehat{\lambda}_{p}(t) \rightarrow \lambda_{p}$ and $\widehat{\lambda}_{D}(t) \rightarrow \lambda_{D}$ with probability 1 as $t \rightarrow \infty$.

Proof. The proof for $\widehat{\lambda}_{p}(t)$ follows immediately from Lemma 3 and [3, $\S 24$, Theorem 1]. Thus it is sufficient to check that $\tilde{\lambda}_{D}(t):=t^{-1} \sum_{k=2}^{\infty}(k-1) \nu(t,\{k\}) \rightarrow \lambda_{p} \lambda_{D}$ with probability 1 as $t \rightarrow \infty$. Note that

$$
Y(t):=\sum_{k=1}^{\infty}(k-1) \nu(t,\{k\})
$$

is a stochastically continuous homogeneous process with independent increments such that

$$
\mathrm{E} Y(1)=\sum_{k=2}^{\infty}(k-1) \lambda_{p} \exp \left\{-\lambda_{D}\right\} \frac{\left(\lambda_{D}\right)^{k-1}}{(k-1) !}=\lambda_{p} \lambda_{D}
$$

The rest of the proof follows again from [3, $\S 24$, Theorem 1].

Further we evaluate the covariance matrix of the estimates $\widehat{\lambda}_{p}(t)$ and $\widehat{\lambda}_{D}(t)$.

\section{Lemma 4.}

$$
\begin{gathered}
\mathrm{E}\left(\widehat{\lambda}_{p}(t)-\lambda_{p}\right)^{2}=\frac{\lambda_{p}}{t}, \quad \mathrm{E}\left(\widehat{\lambda}_{p}(t)-\lambda_{p}\right)\left(\widehat{\lambda}_{D}(t)-\lambda_{D}(t)\left(1-e^{-\lambda_{p} t}\right)\right)=\lambda_{p} \lambda_{D} e^{-\lambda_{p} t}, \\
\mathrm{E}\left(\widehat{\lambda}_{D}(t)-\lambda_{D}\left(1-e^{-\lambda_{p} t}\right)\right)^{2}=\lambda_{D} e^{-\lambda_{p} t} \int_{0}^{\lambda_{p} t} \frac{e^{x}-1}{x} d x+\lambda_{D}^{2} e^{-\lambda_{p} t}\left(1-e^{-\lambda_{p} t}\right) \\
\leq \frac{\lambda_{D}}{\lambda_{p} t}+\frac{\lambda_{D}^{2}}{e^{\lambda_{p} t}} .
\end{gathered}
$$

Corollary. $\hat{\lambda}_{p}(t) \rightarrow \lambda_{p}$ and $\widehat{\lambda}_{D}(t) \rightarrow \lambda_{D}$ in the mean-square sense, and these estimates are asymptotically uncorrelated. 
Proof of Lemma 4. The proof is clear for $\widehat{\lambda}_{p}(t)$, since $\nu(t,[1, \infty))$ has the Poisson distribution with parameter $\lambda_{p} t$. Further,

$$
\begin{aligned}
& \mathrm{E}\left(\widehat{\lambda}_{p}(t)-\lambda_{p}\right)\left(\widehat{\lambda}_{D}(t)-\lambda_{D}\left(1-e^{-\lambda_{p} t}\right)\right) \\
& \quad=\frac{1}{t} \mathrm{E}\left(\sum_{k=2}^{\infty}(k-1) \nu(t,\{k\})\right)-\lambda_{p} \lambda_{D}\left(1-e^{-\lambda_{p} t}\right) \\
& \quad=\frac{1}{t} \sum_{k=2}^{\infty}(k-1) \alpha_{k}-\lambda_{p} \lambda_{D}\left(1-e^{-\lambda_{p} t}\right)=\lambda_{p} \lambda_{D} e^{-\lambda_{p} t}
\end{aligned}
$$

and

$$
\begin{gathered}
\mathrm{E}\left(\frac{\nu(t,\{k\})}{\nu(t,[1, \infty))}\right)^{2}=e^{-\gamma}\left(\alpha_{k}^{2} \sum_{n=0}^{\infty} \frac{\gamma^{n}}{n !(n+2)^{2}}+\alpha_{k} \sum_{n=0}^{\infty} \frac{\gamma^{n}}{n !(n+1)^{2}}\right) \\
\mathrm{E} \frac{\nu(t,\{k\}) \nu(t,\{l\})}{\nu^{2}(t,[1, \infty))}=e^{-\gamma} \sum_{n=0}^{\infty} \frac{\gamma^{n}}{n !(n+2)^{2}}
\end{gathered}
$$

Therefore

$$
\begin{aligned}
\mathrm{E}\left(\widehat{\lambda}_{D}(t)\right)^{2} & =\lambda_{D}^{2}\left(1-e^{-\gamma}\right)+\lambda_{D} e^{-\gamma} \sum_{n=0}^{\infty} \frac{\gamma^{n+1}}{(n+1) !(n+1)} \\
& =\lambda_{D}^{2}\left(1-e^{-\gamma}\right)+\lambda_{D} e^{-\gamma} \int_{0}^{\gamma} \frac{e^{t}-1}{t} d t
\end{aligned}
$$

whence the proof follows.

Denote by $I$ the unit $2 \times 2$ matrix and by $N(0, I)$ the Gaussian distribution with zero mean and covariance matrix $I$. The symbol " $\Rightarrow$ " stands for the weak convergence in distribution.

Theorem 3. The central limit theorem holds in the following sense:

$$
\left(\sqrt{\frac{t}{\lambda_{p}}}\left(\widehat{\lambda}_{p}(t)-\lambda_{p}\right), \sqrt{\frac{t \lambda_{p}}{\lambda_{D}}}\left(\widehat{\lambda}_{D}(t)-\lambda_{D}\right)\right) \Rightarrow N(0, I), \quad t \rightarrow \infty .
$$

Moreover,

$$
\begin{array}{lr}
\sup _{\varepsilon \leq \lambda_{p}, \lambda_{D} \leq \varepsilon^{-1}} \mid \operatorname{Eexp}\left\{i \lambda_{1} \sqrt{\frac{t}{\lambda_{p}}}\left(\hat{\lambda}_{p}(t)-\lambda_{p}\right)+i \lambda_{2} \sqrt{\frac{t \lambda_{p}}{\lambda_{D}}}\left(\widehat{\lambda}_{D}(t)-\lambda_{D}\right)\right\} \\
\leq C \varepsilon^{-r} t^{-1 / 2} & -\exp \left\{-\frac{\lambda_{1}^{2}}{2}-\frac{\lambda_{2}^{2}}{2}\right\} \mid
\end{array}
$$

for all $\lambda_{1}, \lambda_{2} \in \mathbf{R}$, for some $r>0$ and constant $C$ independent of $\varepsilon$ and $t$. Inequality (2) means that the convergence of the characteristic functions is uniform on any rectangle,

$$
\left(\lambda_{p}, \lambda_{D}\right) \in K_{\varepsilon}=\left[\varepsilon, \frac{1}{\varepsilon}\right]^{2}, \quad 0<\varepsilon<1 .
$$


Proof. For all $\lambda_{1}, \lambda_{2} \in \mathbf{R}$,

$$
\begin{aligned}
\xi:= & \lambda_{1} \sqrt{\frac{t}{\lambda_{p}}}\left(\widehat{\lambda}_{p}(t)-\lambda_{p}\right)+\lambda_{2} \sqrt{\frac{t \lambda_{p}}{\lambda_{D}}}\left(\widehat{\lambda}_{D}(t)-\lambda_{D}\right) \\
= & \lambda_{1} \sqrt{\frac{t}{\lambda_{p}}}\left(\frac{\nu(t,[1, \infty))}{t}-\lambda_{p}\right)+\lambda_{2} \sqrt{\frac{t \lambda_{p}}{\lambda_{D}}}\left(\frac{\sum_{k=1}^{\infty}(k-1) \nu(t,\{k\})}{\lambda_{D} \nu(t,[1, \infty))}-\lambda_{D}\right) \\
= & \lambda_{1} \frac{1}{\sqrt{t \lambda_{p}}} \sum_{k=1}^{\infty} \nu(t,\{k\})-\lambda_{1} \sqrt{t \lambda_{p}}+\lambda_{2} \sqrt{\frac{t \lambda_{p}}{\lambda_{D}}} \cdot \frac{\sum_{k=1}^{\infty}\left(k-1-\lambda_{D}\right) \nu(t,\{k\})}{\nu(t,[1, \infty))} \\
= & \frac{\lambda_{1}}{\sqrt{t \lambda_{p}}} \sum_{k=1}^{\infty} \nu(t,\{k\})+\lambda_{2} \frac{1}{\sqrt{t \lambda_{p} \lambda_{D}}} \sum_{k=1}^{\infty}\left(k-1-\lambda_{D}\right) \nu(t,\{k\}) \\
& +\lambda_{2} \sqrt{\frac{t}{\lambda_{p} \lambda_{D}}} \cdot \frac{\sum_{k=1}^{\infty}\left(k-1-\lambda_{D}\right) \nu(t,\{k\})}{\nu(t,[1, \infty))} \cdot\left(\lambda_{p}-\frac{\nu(t,[1, \infty))}{t}\right)-\lambda_{1} \sqrt{t \lambda_{p}} .
\end{aligned}
$$

Consider the term

$$
\begin{aligned}
\eta & :=\lambda_{2} \sqrt{\frac{t}{\lambda_{p} \lambda_{D}}} \cdot \frac{\sum_{k=1}^{\infty}\left(k-1-\lambda_{D}\right) \nu(t,\{k\})}{\nu(t,[1, \infty))} \cdot\left(\lambda_{p}-\frac{\nu(t,[1, \infty))}{t}\right) \\
& =\lambda_{2} \sqrt{\frac{t}{\lambda_{p} \lambda_{D}}} \cdot\left(\widehat{\lambda}_{D}(t)-\lambda_{D}\right) \cdot\left(\lambda_{p}-\widehat{\lambda}_{p}(t)\right) .
\end{aligned}
$$

Taking Lemma 4 into account, we obtain that

$$
\begin{aligned}
\sup _{\left(\lambda_{p}, \lambda_{D}\right) \in K_{\varepsilon}} \mathrm{E}|\eta| & \leq \sup _{\left(\lambda_{p}, \lambda_{D}\right) \in K_{\varepsilon}} \frac{\lambda_{2} \sqrt{t}}{\sqrt{\lambda_{p} \lambda_{D}}} \cdot \sqrt{\frac{\lambda_{p}}{t}} \cdot \sqrt{\frac{\lambda_{D}}{\lambda_{p} t}+\frac{\lambda_{D}^{2}}{e^{\lambda_{p} t}}+\frac{\lambda_{D}^{2}}{e^{2 \lambda_{p} t}}} \\
& \leq \frac{\lambda_{2}}{\sqrt{t}} \sqrt{\frac{1}{\varepsilon}+\frac{t}{\varepsilon^{2} e^{\varepsilon t}}+\frac{t}{\varepsilon^{2} e^{2 \varepsilon t}}} \leq \frac{\lambda_{2}}{\sqrt{t}} \sqrt{\frac{1}{\varepsilon}+\frac{3}{2 \varepsilon^{3}}} .
\end{aligned}
$$

Now we estimate the difference

$$
\begin{aligned}
& \left|\mathrm{E} e^{i \xi}-e^{-\lambda_{1}^{2} / 2-\lambda_{2}^{2} / 2}\right| \\
& \quad \leq \mathrm{E}|\eta|+\left|\mathrm{E} \exp \left\{i \sum_{k=1}^{\infty} a_{k} \nu(t,\{k\})-i \lambda_{1} \sqrt{t \lambda_{p}}\right\}-e^{-\lambda_{1}^{2} / 2-\lambda_{2}^{2} / 2}\right|,
\end{aligned}
$$

where

$$
a_{k}=\frac{\lambda_{1}}{\sqrt{t \lambda_{p}}}+\frac{\lambda_{2}}{\sqrt{t \lambda_{p} \lambda_{D}}}\left(k-1-\lambda_{D}\right) .
$$

It is evident that

$$
a:=\exp \left\{-i \lambda_{1} \sqrt{t \lambda_{p}}\right\} \cdot \operatorname{E} \exp \left\{i \sum_{k=1}^{\infty} a_{k} \nu(t,\{k\})\right\}=\alpha \cdot \prod_{k=1}^{\infty} \mathrm{E} \exp \left\{i a_{k} \nu(t,\{k\})\right\},
$$

where $\alpha=\exp \left\{-i \lambda_{1} \sqrt{t \lambda_{p}}\right\}$. Further, $\nu(t,\{k\})$ are Poisson random variables, thus

$$
\mathrm{E} \exp \left\{i a_{k} \nu(t,\{k\})\right\}=\exp \left\{\alpha_{k}\left[\exp \left\{i a_{k}\right\}-1\right]\right\},
$$

whence

$$
a=\alpha \cdot \exp \left\{\sum_{k=1}^{\infty} \alpha_{k} \cdot \exp \left\{i a_{k}\right\}-\lambda_{p} t\right\}
$$


Consider the series under the exponential,

$$
\begin{aligned}
& b:=\sum_{k=1}^{\infty} \alpha_{k} \exp \left\{i a_{k}\right\}=\sum_{k=1}^{\infty} \lambda_{p} t \frac{\left(\lambda_{D}\right)^{k-1}}{(k-1) !} e^{-\lambda_{D}} \exp \left\{i\left(\frac{\lambda_{1}-\lambda_{2} \sqrt{\lambda_{D}}}{\sqrt{\lambda_{p} t}}\right)+i \frac{\lambda_{2}(k-1)}{\sqrt{\lambda_{p} \lambda_{D} t}}\right\} \\
&=\lambda_{p} t \exp \left\{i\left(\frac{\lambda_{1}-\lambda_{2} \sqrt{\lambda_{D}}}{\sqrt{\lambda_{p} t}}\right)\right\} e^{-\lambda_{D}} \cdot \sum_{k=1}^{\infty} \frac{\left(\lambda_{D}\right)^{k-1}}{(k-1) !} \exp \left\{\frac{i \lambda_{2}}{\sqrt{\lambda_{p} \lambda_{D} t}}(k-1)\right\} \\
&=\lambda_{p} t \exp \left\{i\left(\frac{\lambda_{1}-\lambda_{2} \sqrt{\lambda_{D}}}{\sqrt{\lambda_{p} t}}\right)\right\} e^{-\lambda_{D}} \cdot \exp \left\{\lambda_{D} \cdot \exp \left(\frac{i \lambda_{2}}{\sqrt{\lambda_{p} \lambda_{D} t}}\right)\right\} \\
&=\lambda_{p} t \exp \left\{\lambda_{D}\left(\cos \frac{\lambda_{2}}{\sqrt{\lambda_{p} \lambda_{D} t}}-1\right)+i\left(\frac{\lambda_{1}-\lambda_{2} \sqrt{\lambda_{D}}}{\sqrt{\lambda_{p} t}}+\lambda_{D} \sin \frac{\lambda_{2}}{\sqrt{\lambda_{p} \lambda_{D} t}}\right)\right\} \\
&=\lambda_{p} t \exp \left\{\lambda_{D}\left(\cos \frac{\lambda_{2}}{\sqrt{\lambda_{p} \lambda_{D} t}}-1\right)\right\} \cdot\left[\cos \left(\frac{\lambda_{1}-\lambda_{2} \sqrt{\lambda_{D}}}{\sqrt{\lambda_{p} t}}+\lambda_{D} \sin \frac{\lambda_{2}}{\sqrt{\lambda_{p} \lambda_{D} t}}\right)\right. \\
&\left.+i \sin \left(\frac{\lambda_{1}-\lambda_{2} \sqrt{\lambda_{D}}}{\sqrt{\lambda_{p} t}}+\lambda_{D} \sin \frac{\lambda_{2}}{\sqrt{\lambda_{p} \lambda_{D} t}}\right)\right] .
\end{aligned}
$$

We treat the real and imaginary parts separately by using the Taylor formula,

$$
\begin{aligned}
\operatorname{Im} b= & \lambda_{p} t\left(1-\frac{\lambda_{D} \lambda_{2}^{2}}{2 \lambda_{p} \lambda_{D} t}+O_{\varepsilon}\left(\frac{1}{t^{2}}\right)\right) \\
& \times\left(\frac{\lambda_{1}-\lambda_{2} \sqrt{\lambda_{D}}}{\sqrt{\lambda_{p} t}}+\lambda_{D} \frac{\lambda_{2}}{\sqrt{\lambda_{p} \lambda_{D} t}}+O_{\varepsilon}\left(\frac{1}{t^{3 / 2}}\right)\right) \\
= & \lambda_{1} \sqrt{\lambda_{p} t}+O_{\varepsilon}\left(\frac{1}{t^{1 / 2}}\right),
\end{aligned}
$$

where

$$
\left|O_{\varepsilon}\left(t^{-k}\right)\right| \leq C \varepsilon^{-r} t^{-k} \quad \text { as } t \rightarrow \infty
$$

for some $r>0$. Further

$$
\begin{aligned}
\operatorname{Re} b= & \lambda_{p} t\left(1-\frac{\lambda_{D}}{2} \cdot \frac{\lambda_{2}^{2}}{\lambda_{p} \lambda_{D} t}+O_{\varepsilon}\left(\frac{1}{t^{2}}\right)\right) \\
& \times\left(1-\frac{1}{2}\left(\frac{\lambda_{1}-\lambda_{2} \sqrt{\lambda_{D}}}{\sqrt{\lambda_{p} t}}+\lambda_{D} \sin \frac{\lambda_{2}}{\sqrt{\lambda_{p} \lambda_{D} t}}\right)^{2}+O_{\varepsilon}\left(\frac{1}{t^{2}}\right)\right) \\
= & \lambda_{p} t-\frac{\lambda_{1}^{2}}{2}-\frac{\lambda_{2}^{2}}{2}+O_{\varepsilon}\left(\frac{1}{t^{1 / 2}}\right) .
\end{aligned}
$$

Substituting expansions (6) and (7) into (5), we obtain

$$
a=\exp \left\{-\frac{\lambda_{1}^{2}}{2}-\frac{\lambda_{2}^{2}}{2}+O_{\varepsilon}\left(\frac{1}{t^{1 / 2}}\right)\right\}=\exp \left\{-\frac{\lambda_{1}^{2}}{2}-\frac{\lambda_{2}^{2}}{2}\right\}\left(1+O_{\varepsilon}\left(\frac{1}{t^{1 / 2}}\right)\right) .
$$

Using bounds (3), (4), and (8) we get

$$
\sup _{\varepsilon \leq \lambda_{p}, \lambda_{D} \leq \varepsilon^{-1}}\left|\operatorname{E} \exp \{i \xi\}-\exp \left\{-\frac{\lambda_{1}^{2}}{2}-\frac{\lambda_{2}^{2}}{2}\right\}\right|=O_{\varepsilon}\left(\frac{1}{t^{1 / 2}}\right)
$$

whence the theorem follows. 
O. G. KUKUSH AND YU. S. MISHURA

\section{LOCAL ASYMPTOTIC NORMALITY OF ESTIMATES}

Let $\Theta=(0, \infty) \times(0, \infty)$ and note that $\theta=\left(\lambda_{p}, \lambda_{D}\right) \in \Theta$. Denote the measure $P_{\lambda_{p}, \lambda_{D}}(t)$ (see Section 2) by $P_{\theta}(t)$. According to [5, Definition 2.1], a family of measures $P_{\theta}(t)$ is called locally asymptotically normal at the point $\theta \in \Theta$ as $t \rightarrow \infty$ if

$$
Z_{t, \theta}(u)=\frac{d P_{\theta+A(t, \theta) u}(t)}{d P_{\theta}(t)}\left(X_{t}\right)=\exp \left\{u_{1} \xi_{1}^{t, \theta}+u_{2} \xi_{2}^{t, \theta}-\frac{1}{2} u_{1}^{2}-\frac{1}{2} u_{2}^{2}+\xi_{t}(u, \theta)\right\}
$$

for some nonsingular $2 \times 2$ matrix $A(t, \theta)$ and for all vectors $u \in \mathbf{R}^{2}$, where

$$
\left(\xi_{1}^{t, \theta}, \xi_{2}^{t, \theta}\right) \Rightarrow N(0, I) \quad \text { as } t \rightarrow \infty
$$

with respect to the measure $P_{\theta}(t)$, and

$$
\xi_{t}(u, \theta) \stackrel{P_{\theta}(t)}{\longrightarrow} \longrightarrow 0 \quad \text { as } t \rightarrow \infty
$$

for all $u \in \mathbf{R}^{2}$. We say briefly that in this case the LAN property holds for the family $P_{\theta}(t)$ at the point $\theta$ as $t \rightarrow \infty$.

Theorem 4. A family $P_{\theta}(t)$ is locally asymptotically normal at any point $\theta \in \Theta$ as $t \rightarrow \infty$, where the matrix $A(t, \theta)$ is of the form

$$
A(t, \theta)=(2 t)^{-1 / 2}\left(\begin{array}{cc}
\lambda_{p}^{1 / 2} & \lambda_{p}^{1 / 2} \\
\lambda_{p}^{-1 / 2} \lambda_{D}^{1 / 2} & -\lambda_{p}^{-1 / 2} \lambda_{D}^{1 / 2}
\end{array}\right) .
$$

Proof. We use the likelihood ratio obtained in Lemma 2:

$$
\begin{aligned}
Z_{t, \theta}(u) & =\frac{d P_{\theta+A(t) u}(t)}{d P_{1,1}(t)} \cdot\left(\frac{d P_{\theta}(t)}{d P_{1,1}(t)}\right)^{-1} \\
& =\exp \left\{\sum_{k=1}^{\infty}\left(\ln \frac{\lambda_{p}^{1}}{\lambda_{p}^{2}}-\left(\lambda_{D}^{1}-\lambda_{D}^{2}\right)+(k-1) \ln \frac{\lambda_{D}^{1}}{\lambda_{D}^{2}}\right) \nu(t,\{k\})-t\left(\lambda_{p}^{1}-\lambda_{p}^{2}\right)\right\},
\end{aligned}
$$

where

and

$$
\lambda_{p}^{1}=\lambda_{p}+\sqrt{\frac{\lambda_{p}}{2 t}} u_{1}+\sqrt{\frac{\lambda_{p}}{2 t}} u_{2}, \quad \lambda_{p}^{2}=\lambda_{p}
$$

$$
\lambda_{D}^{1}=\lambda_{D}+\sqrt{\frac{\lambda_{D}}{2 \lambda_{p} t}} u_{1}-\sqrt{\frac{\lambda_{D}}{2 \lambda_{p} t}} u_{2}, \quad \lambda_{D}^{2}=\lambda_{D} .
$$

Substituting $\lambda_{p}^{i}$ and $\lambda_{D}^{i}, i=1,2$, into (10) we obtain

$$
\begin{aligned}
Z_{t, \theta}(u)=\exp \left\{\nu(t,[1, \infty))\left[\ln \left(1+\frac{a_{1} u_{1}+a_{2} u_{2}}{\lambda_{p}}\right)-\left(a_{3} u_{1}+a_{4} u_{2}\right)\right]\right. \\
\left.\quad+\ln \left(1+\frac{a_{3} u_{1}+a_{4} u_{2}}{\lambda_{D}}\right) \cdot \sum_{k=2}^{\infty}(k-1) \nu(t,\{k\})-t\left(a_{1} u_{1}+a_{2} u_{2}\right)\right\},
\end{aligned}
$$

where $a_{1}=a_{2}=\left(\lambda_{p} /(2 t)\right)^{1 / 2}$ and $a_{3}=-a_{4}=\left(\lambda_{D} /\left(2 \lambda_{p} t\right)\right)^{1 / 2}$. Put

$$
\begin{gathered}
\alpha=\ln \left(1+\left(a_{3} u_{1}+a_{4} u_{2}\right) / \lambda_{D}\right), \\
\beta=\ln \left(1+\left(a_{1} u_{1}+a_{2} u_{2}\right) / \lambda_{p}\right)-a_{3} u_{1}-a_{4} u_{2} .
\end{gathered}
$$

Then

$$
Z_{t, \theta}(u)=\exp \left\{\sum_{k=1}^{\infty}((k-1) \alpha+\beta) \nu(t,\{k\})-t\left(a_{1} u_{1}+a_{2} u_{2}\right)\right\}
$$


Consider an auxiliary random variable

$$
\xi_{t}:=\sum_{k=1}^{\infty}((k-1) \alpha+\beta) \nu(t,\{k\})-\lambda_{p} \lambda_{D} \alpha t-\lambda_{p} \beta t
$$

and evaluate its characteristic function $\operatorname{Exp}\left\{i \lambda \xi_{t}\right\}$. Similarly to the proof of Theorem 3,

$$
\begin{aligned}
\mathrm{E} \exp \left\{i \lambda \xi_{t}\right\} & =\exp \left\{\lambda \exp \{i \beta\} \lambda_{p} t e^{-\lambda_{D}} \exp \left\{\lambda_{D} e^{i \alpha}\right\}-\lambda_{p} t\right\} \cdot \exp \left\{i \lambda\left(-\lambda_{p} \lambda_{D} \alpha t-\lambda_{p} \beta t\right)\right\} \\
& :=\exp \{\delta\} .
\end{aligned}
$$

Moreover

$$
\begin{aligned}
\operatorname{Im} \delta= & \lambda \cdot \lambda_{p} t \exp \left\{\lambda_{D}(\cos \alpha-1)\right\} \cdot \sin \left(\beta+\lambda_{D} \sin \alpha\right)-\lambda \lambda_{p} \lambda_{D} \alpha t-\lambda \lambda_{p} \beta t \\
= & \lambda \lambda_{p} t\left(1-\lambda_{D}\left(\frac{a_{3} u_{1}+a_{4} u_{2}}{\lambda_{D}}\right)^{2}+o\left(\frac{1}{t}\right)\right)\left(\frac{a_{1} u_{1}+a_{2} u_{2}}{\lambda_{p}}+o\left(\frac{1}{t^{1 / 2}}\right)\right) \\
& -\lambda t\left(a_{1} u_{1}+a_{2} u_{2}\right)+o\left(\frac{1}{t^{1 / 2}}\right) \\
= & o\left(\frac{1}{t^{1 / 2}}\right)
\end{aligned}
$$

as $t \rightarrow \infty$, and

$$
\begin{aligned}
\operatorname{Re} \delta= & \lambda \cdot \lambda_{p} t \exp \left\{\lambda_{D}(\cos \alpha-1)\right\} \cdot \cos \left(\beta+\lambda_{D} \sin \alpha\right) \\
= & \lambda \lambda_{p} t\left(1-\frac{\lambda_{D}}{2}\left(\frac{a_{3} u_{1}+a_{4} u_{2}}{\lambda_{D}}\right)^{2}\right)\left(1-\frac{1}{2}\left(\frac{a_{1} u_{1}+a_{2} u_{2}}{\lambda_{p}}+o\left(\frac{1}{t^{1 / 2}}\right)\right)^{2}\right) \\
& -\lambda_{p} t+o\left(\frac{1}{t^{1 / 2}}\right) \\
= & -\frac{\lambda \lambda_{p} t}{2 \lambda_{D}}\left(a_{3} u_{1}+a_{4} u_{2}\right)^{2}-\frac{\lambda t}{2 \lambda_{p}}\left(a_{1} u_{1}+a_{2} u_{2}\right)^{2}+o\left(\frac{1}{t^{1 / 2}}\right) \\
= & -\frac{\lambda}{4}\left(u_{1}-u_{2}\right)^{2}-\frac{\lambda}{4}\left(u_{1}+u_{2}\right)^{2}=-\frac{\lambda}{2} u_{1}^{2}-\frac{\lambda}{2} u_{2}^{2}+o\left(\frac{1}{t^{1 / 2}}\right)
\end{aligned}
$$

as $t \rightarrow \infty$. Thus

$$
\xi_{t} \Rightarrow u^{T} N(0, I)
$$

Now we use the asymptotic expansions again and obtain

$$
\begin{aligned}
Z_{t, \theta}(u) & =\exp \left\{\xi_{t}-t\left(a_{1} u_{1}+a_{2} u_{2}\right)+\lambda_{p} \lambda_{D} \alpha t+\lambda_{p} \beta t\right\} \\
& =\exp \left\{\xi_{t}-\frac{\lambda_{p} t}{\lambda_{D}}\left(a_{3} u_{1}+a_{4} u_{2}\right)^{2}-\lambda_{p} t\left(a_{1} u_{1}+a_{2} u_{2}\right)^{2}+\left(\frac{1}{t^{1 / 2}}\right)\right\} \\
& =\exp \left\{\xi_{t}-\frac{u_{1}^{2}}{2}-\frac{u_{2}^{2}}{2}+o\left(\frac{1}{t^{1 / 2}}\right)\right\} .
\end{aligned}
$$

Relation (9) follows from (11) and (12) by setting

$$
\begin{aligned}
\xi_{1}^{t, \theta} & =\sum_{k=1}^{\infty}\left[(k-1) \frac{1}{\sqrt{2 \lambda_{p} \lambda_{D} t}}+\frac{1}{\sqrt{2 \lambda_{p} t}}-\sqrt{\frac{\lambda_{D}}{2 \lambda_{p} t}}\right] \cdot \nu(t,\{k\})-\sqrt{\frac{\lambda_{p} t}{2}}, \\
\xi_{2}^{t, \theta} & =\sum_{k=1}^{\infty}\left[-(k-1) \frac{1}{\sqrt{2 \lambda_{p} \lambda_{D} t}}+\frac{1}{\sqrt{2 \lambda_{p} t}}+\sqrt{\frac{\lambda_{D}}{2 \lambda_{p} t}}\right] \cdot \nu(t,\{k\})-\sqrt{\frac{\lambda_{p} t}{2}} .
\end{aligned}
$$

The theorem is proved. 


\section{Asymptotic EFFiCIENCY OF THE EStimates}

First we evaluate the inverse matrix of $A(t, \theta)$. Evidently,

$$
A^{-1}(t, \theta)=\sqrt{\frac{t}{2}}\left(\begin{array}{cc}
\frac{1}{\sqrt{\lambda_{p}}} & \sqrt{\frac{\lambda_{p}}{\lambda_{D}}} \\
\frac{1}{\sqrt{\lambda_{p}}} & -\sqrt{\frac{\lambda_{p}}{\lambda_{D}}}
\end{array}\right) .
$$

According to [5], an estimate $\left\{\theta_{t}, t>0\right\}$ of a parameter $\theta$ is called asymptotically efficient for a cost function $w\left(A^{-1}(t, \theta) x\right)$ at the point $\theta$ if the LAN property holds and

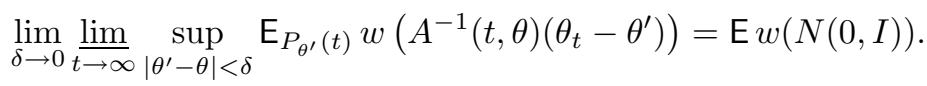

Note that, under the LAN property,

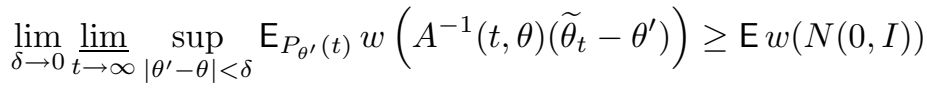

for all estimates $\widetilde{\theta}_{t}$ and cost functions $w \in W$, where $W$ is a class of functions defined on $\Theta$ and such that

1) $w(u) \geq 0, w(0)=0, w$ is Borel and continuous at zero and is not identically zero;

2) $w(u)=w(-u)$

3) the set $\{u: w(u)<c\}$ is convex for every $c>0$.

Theorem 5. The estimate $\theta_{t}:=\left(\widehat{\lambda}_{p}(t), \widehat{\lambda}_{D}(t)\right)$ of the parameter $\theta:=\left(\lambda_{p}, \lambda_{D}\right)$ is asymptotically efficient on any rectangle $\theta \in K_{\varepsilon}=[\varepsilon, 1 / \varepsilon]^{2}$ for the cost function

$$
w\left(A^{-1}(t, \theta) x\right) \in W_{p},
$$

where $W_{p} \subset W$ is the subclass of $W$ consisting of functions with a polynomial dominant.

Proof. To prove the asymptotic efficiency of the estimate $\theta_{t}:=\left(\widehat{\lambda}_{p}(t), \widehat{\lambda}_{D}(t)\right)$ of the parameter $\theta:=\left(\lambda_{p}, \lambda_{D}\right)$ we use Theorem 1.3 in [5]. According to this result the asymptotic efficiency of the estimate $\theta_{t}$ holds if the following conditions $(\mathrm{A})-(\mathrm{C})$ are satisfied:

(A) there exists the limit

$$
\lim _{t \rightarrow \infty} A^{-1}\left(t, \theta_{2}\right) A\left(t, \theta_{1}\right)=B\left(\theta_{1}, \theta_{2}\right)
$$

where the convergence is uniform in $\theta_{1}, \theta_{2} \in K_{\varepsilon}$;

(B) uniformly in $\theta \in K_{\varepsilon}$,

$$
\zeta_{t}(\theta):=A^{-1}(t, \theta)\left(\theta_{t}-\theta\right) \Rightarrow N(0, I) \quad \text { as } t \rightarrow \infty
$$

with respect to the measure $P_{\theta}(t)$;

(C) for every $N>0$, the family of random variables

$$
\left|A^{-1}(t, \theta)\left(\theta_{t}-\theta\right)\right|^{N}
$$

is $P_{\theta}(t)$-integrable uniformly in $\theta \in K_{\varepsilon}, t \geq t_{0}(N)$.

To check condition (A) we write

$$
\begin{aligned}
& A^{-1}\left(t, \theta_{2}\right) A\left(t, \theta_{1}\right)=\left(\begin{array}{cc}
\sqrt{\frac{t}{2 \lambda_{p}^{2}}} & \sqrt{\frac{\lambda_{p}^{2} t}{2 \lambda_{D}^{2}}} \\
\sqrt{\frac{t}{2 \lambda_{p}^{2}}} & -\sqrt{\frac{\lambda_{p}^{2} t}{2 \lambda_{D}^{2}}}
\end{array}\right) \cdot\left(\begin{array}{cc}
\sqrt{\frac{\lambda_{p}^{1}}{2 t}} & \sqrt{\frac{\lambda_{p}^{1}}{2 t}} \\
\sqrt{\frac{\lambda_{D}^{1}}{2 \lambda_{p}^{1} t}} & -\sqrt{\frac{\lambda_{D}^{1}}{2 \lambda_{p}^{1} t}}
\end{array}\right) \\
& =\left(\begin{array}{ll}
\frac{1}{2} \sqrt{\frac{\lambda_{p}^{1}}{\lambda_{p}^{2}}}+\frac{1}{2} \sqrt{\frac{\lambda_{p}^{2} \lambda_{D}^{1}}{\lambda_{p}^{1} \lambda_{D}^{2}}} & \frac{1}{2} \sqrt{\frac{\lambda_{p}^{1}}{\lambda_{p}^{2}}}-\frac{1}{2} \sqrt{\frac{\lambda_{p}^{2} \lambda_{D}^{1}}{\lambda_{p}^{1} \lambda_{D}^{2}}} \\
\frac{1}{2} \sqrt{\frac{\lambda_{p}^{1}}{\lambda_{p}^{2}}}-\frac{1}{2} \sqrt{\frac{\lambda_{p}^{2} \lambda_{D}^{1}}{\lambda_{p}^{1} \lambda_{D}^{2}}} & \frac{1}{2} \sqrt{\frac{\lambda_{p}^{1}}{\lambda_{p}^{2}}}+\frac{1}{2} \sqrt{\frac{\lambda_{p}^{2} \lambda_{D}^{1}}{\lambda_{p}^{1} \lambda_{D}^{2}}}
\end{array}\right) .
\end{aligned}
$$


The matrix on the right-hand side does not depend on $t$, and condition (A) holds, indeed.

Now we check condition (B). By Theorem 7 [5] p. 480], the uniform weak convergence of $\zeta_{t}(\theta)$ to $N(0, I)$ follows from the conditions

$\left.\left(\mathrm{B}^{\prime}\right), 1\right) \sup _{\theta \in K_{\varepsilon}} \mathrm{P}_{\theta}\left\{\left|\zeta_{t}(\theta)\right|>C\right\} \rightarrow 0$ as $C \rightarrow \infty$;

2) for $\lambda=\left(\lambda_{1}, \lambda_{2}\right)$,

$$
\mathrm{E}_{P_{\theta}(t)} \exp \left\{i\left(\zeta_{t}(\theta), \lambda\right)\right\} \rightarrow \exp \left\{-\frac{\lambda_{1}^{2}}{2}-\frac{\lambda_{2}^{2}}{2}\right\}, \quad t \rightarrow \infty,
$$

uniformly in $\theta \in K_{\varepsilon}$.

In order to check condition $\left.\left(\mathrm{B}^{\prime}\right), 1\right)$ we consider the vector $\zeta_{t}(\theta)=\left(\zeta_{t}^{1}(\theta), \zeta_{t}^{2}(\theta)\right)$, where

$$
\begin{aligned}
& \zeta_{t}^{1}(\theta)=\sqrt{\frac{t}{\lambda_{p}}}\left(\widehat{\lambda}_{p}(t)-\lambda_{p}\right)+\sqrt{\frac{t \lambda_{p}}{\lambda_{D}}}\left(\widehat{\lambda}_{D}(t)-\lambda_{D}\right), \\
& \zeta_{t}^{2}(\theta)=\sqrt{\frac{t}{\lambda_{p}}}\left(\widehat{\lambda}_{p}(t)-\lambda_{p}\right)-\sqrt{\frac{t \lambda_{p}}{\lambda_{D}}}\left(\widehat{\lambda}_{D}(t)-\lambda_{D}\right) .
\end{aligned}
$$

Using Lemma 4 we estimate the moments of the right-hand side of (14):

$$
\mathrm{E}\left|\sqrt{\frac{t}{\lambda_{p}}}\left(\widehat{\lambda}_{p}(t)-\lambda_{p}\right)\right|^{2}=1, \quad \mathrm{E}\left|\sqrt{\frac{t \lambda_{p}}{\lambda_{D}}}\left(\widehat{\lambda}_{D}(t)-\lambda_{D}\right)\right|^{2} \leq 1+\frac{3 \lambda_{D}}{e^{\lambda_{p} t}} \leq 1+\frac{3}{\varepsilon} .
$$

It is easy to see that inequality $\left.\left(\mathrm{B}^{\prime}\right), 1\right)$ follows from $(15)$. Now we apply inequality $(2)$ :

$$
\begin{aligned}
& \mathrm{E}_{P_{\theta}(t)} \exp \left\{i\left(\zeta_{t}(\theta), \lambda\right)\right\}=\mathrm{E}_{P_{\theta}(t)} \exp \left\{i \lambda_{1} \zeta_{t}^{1}(\theta)+i \lambda_{2} \zeta_{t}^{2}(\theta)\right\} \\
& \quad=\mathrm{E}_{P_{\theta}(t)} \exp \left\{i\left(\lambda_{1}+\lambda_{2}\right) \sqrt{\frac{t}{\lambda_{p}}}\left(\widehat{\lambda}_{p}(t)-\lambda_{p}\right)+i\left(\lambda_{1}-\lambda_{2}\right) \sqrt{\frac{t \lambda_{p}}{\lambda_{D}}}\left(\widehat{\lambda}_{D}(t)-\lambda_{D}\right)\right\} \\
& \quad \rightarrow \exp \left\{-\frac{\lambda_{1}^{2}}{2}-\frac{\lambda_{2}^{2}}{2}\right\},
\end{aligned}
$$

where the convergence is uniform on $K_{\varepsilon}$. Therefore condition $\left.\left(\mathrm{B}^{\prime}\right), 2\right)$ holds, whence (B) follows.

Finally we check condition (C). It follows from representation (14) that the following two conditions:

$$
\sup _{\substack{\theta \in K_{\varepsilon} \\ t \geq t_{0}(N)}} \mathrm{E}_{P_{\theta}(t)}\left|\sqrt{\frac{t}{\lambda_{p}}}\left(\widehat{\lambda}_{p}(t)-\lambda_{p}\right)\right|^{2 N} \leq C_{N, \varepsilon}
$$

and

$$
\sup _{\substack{\theta \in K_{\varepsilon} \\ t>t_{0}(N)}} \mathrm{E}_{P_{\theta}(t)}\left|\sqrt{\frac{t \lambda_{p}}{\lambda_{D}}}\left(\widehat{\lambda}_{D}(t)-\lambda_{D}\right)\right|^{2 N} \leq C_{N, \varepsilon}
$$

for all $N \geq 1$ are sufficient for $(\mathrm{C})$, where the constants $C_{N, \varepsilon}$ depend on $N$ and $\varepsilon$. In what follows we omit the subscript $P_{\theta}(t)$. Note that

$$
\mathrm{E}\left|\sqrt{\frac{t}{\lambda_{p}}}\left(\widehat{\lambda}_{p}(t)-\lambda_{p}\right)\right|^{2 N}=\frac{t^{-N}}{\lambda_{p}^{N}} \mathrm{E}\left(\nu(t,[1, \infty))-\lambda_{p} t\right)^{2 N}
$$


Let $t_{0}(N)=1 / \varepsilon$, that is, $\lambda_{p} t \geq 1$. The central moments of the Poisson distribution satisfy the following recurrence formula:

$$
\mu_{k}=\lambda \sum_{l=0}^{k-2} C_{k-1}^{l} \mu_{l}, \quad k \geq 2,
$$

where $\lambda$ is the parameter of the distribution.

Assume that $\lambda>1$ and for all $k \leq l$

$$
\mu_{k} \leq C_{k} \lambda^{(k+1) / 2}
$$

for odd $k$, and

$$
\mu_{k} \leq C_{k} \lambda^{k / 2}
$$

for even $k$. For example, let $l=2 p$ be an even number. Then it follows from (18) that

$$
\begin{aligned}
\mu_{l+1} & =\mu_{2 p+1} \leq \lambda \sum_{\substack{r=0 \\
r \text { is even }}}^{l-1} C_{l}^{r} C_{r} \lambda^{r / 2}+\lambda \sum_{\substack{r=0 \\
r \text { is odd }}}^{l-1} C_{l}^{r} C_{r} \lambda^{(r+1) / 2} \\
& \leq C_{2 p+1} t^{p+1}=C_{2 p+1} \lambda^{((2 p+1)+1) / 2},
\end{aligned}
$$

and similarly

$$
\begin{aligned}
\mu_{l+2} & =\mu_{2 p+2} \leq \lambda \sum_{\substack{r=0 \\
r \text { is even }}}^{l} C_{l+1}^{r} C_{r} \lambda^{r / 2}+\lambda \sum_{\substack{r=0 \\
r \text { is odd }}}^{l} C_{l+1}^{r} C_{r} \lambda^{(r+1) / 2} \\
& \leq C_{2 p+2} \lambda^{p+1}=C_{2 p+2} \lambda^{(2 p+2) / 2} .
\end{aligned}
$$

The case of an odd number $l=2 p+1$ is considered similarly.

Since the assumption is true for $k=1$ and $k=2$, we obtain $\mu_{k} \leq C_{k} \lambda^{(k+1) / 2}$ for odd $k$, and $\mu_{k} \leq C_{k} \lambda^{k / 2}$ for even $k$. In particular,

$$
\frac{t^{-N}}{\lambda_{p}^{N}} \mathrm{E}\left(\nu(t,[1, \infty))-\lambda_{p} t\right)^{2 N} \leq \frac{t^{-N}}{\lambda_{p}^{N}} C_{2 N}\left(\lambda_{p} t\right)^{N} \leq C_{2 N},
$$

whence (16) follows.

In order to prove (17) we write

$$
\begin{aligned}
\mathrm{E}\left|\sqrt{\frac{t \lambda_{p}}{\lambda_{D}}}\left(\hat{\lambda}_{D}(t)-\lambda_{D}\right)\right|^{2 N} & =t^{N}\left(\frac{\lambda_{p}}{\lambda_{D}}\right)^{N} \mathrm{E}\left|\frac{\sum_{k=1}^{\infty}\left(k-1-\lambda_{D}\right) \nu(t,\{k\})}{\nu(t,[1, \infty))}\right|^{2 N} \\
\leq & t^{-N} \varepsilon^{-2 N}\left(\mathrm{E}\left|\sum_{k=1}^{\infty}\left(k-1-\lambda_{D}\right) \nu(t,\{k\})\right|^{4 N}\right)^{1 / 2} \\
& \times\left(\mathrm{E}\left|\frac{t}{\nu(t,[1, \infty)) I}\right|^{4 N}\right)^{1 / 2},
\end{aligned}
$$

where $I=I\{\nu(t,[1, \infty)) \geq 1\}$.

Consider the characteristic function

$$
\begin{aligned}
\varphi(\lambda) & :=\operatorname{E} \exp \left\{i \lambda \sum_{k=1}^{\infty}\left(k-1-\lambda_{D}\right) \nu(t,\{k\})\right\} \\
& =\exp \left\{\lambda_{p} t\left[\exp \left\{\lambda_{D}\left(e^{i \lambda}-1-i \lambda\right)\right\}-1\right]\right\} .
\end{aligned}
$$

The function $\varphi(\lambda)$ can be viewed as the characteristic function of the sum $\sum_{k=0}^{\mu} \psi_{k}$, where

$$
\psi_{k}=\bar{\psi}_{k}-\mathrm{E} \bar{\psi}_{k}
$$


are independent identically distributed centred random variables, $\bar{\psi}_{k}$ has the Poisson distribution with parameter $\lambda_{D}$, and $\mu$ has the Poisson distribution with parameter $\lambda_{p} t$. Therefore

$$
\mathrm{E}\left|\sum_{k=1}^{\infty}\left(k-1-\lambda_{D}\right) \nu(t,\{k\})\right|^{4 N}=\mathrm{E}\left|\sum_{k=0}^{\mu} \psi_{k}\right|^{4 N}=\sum_{r=0}^{\infty} \mathrm{E}\left|\sum_{k=0}^{r} \psi_{k}\right|^{4 N} \frac{\left(\lambda_{p} t\right)^{r} e^{-\lambda_{p} t}}{r !} .
$$

According to the preceding estimates for central moments of the Poisson distribution we obtain

$$
\mathrm{E}\left|\sum_{k=0}^{r} \psi_{k}\right|^{4 N} \leq C_{4 N}\left(\lambda_{D} r\right)^{2 N}
$$

for $\lambda_{D} r>1$, since $\sum_{k=0}^{r} \psi_{k}$ has the Poisson distribution with parameter $r \lambda_{D}$. The same estimates applied to the case of $\lambda_{D} r<1$ imply that $\mathrm{E}\left|\sum_{k=0}^{r} \psi_{k}\right|^{4 N} \leq C_{4 N}$.

Thus

$$
\begin{aligned}
\mathrm{E} \mid \sum_{k=1}^{\infty}( & \left.k-1-\lambda_{D}\right)\left.\nu(t,\{k\})\right|^{4 N} \leq C_{4 N} \sum_{r=0}^{\infty}\left(\left(\lambda_{D} r\right)^{2 N} \vee 1\right) \frac{\left(\lambda_{p} t\right)^{r} \cdot e^{-\lambda_{p} t}}{r !} \\
\leq & C_{4 N} \\
& +C_{4 N} \lambda_{D}^{2 N}\left(\sum_{r=1}^{2 N} \frac{\left(\lambda_{p} t\right)^{r} \cdot e^{-\lambda_{p} t}}{r !}(2 N)^{2 N}\right. \\
& \left.\quad+\sum_{r=2 N+1}^{\infty} \frac{e^{-\lambda_{p} t}\left(\lambda_{p} t\right)^{r-2 N}}{(r-2 N) !}\left(\lambda_{p} t\right)^{2 N} \cdot\left(\frac{2 N+1}{2}\right)^{2 N}\right) \\
\leq & C_{2 N} \lambda_{D}^{2 N}\left((2 N)^{2 N}+\left(\frac{2 N+1}{2}\right)^{2 N} \cdot\left(\lambda_{p} t\right)^{2 N}\right)
\end{aligned}
$$

and

$$
\begin{aligned}
\mathrm{E}\left|\frac{t}{\nu(t,[1, \infty)) I}\right|^{4 N} & =\sum_{r=1}^{\infty}\left(\frac{t}{r}\right)^{4 N} \cdot \frac{e^{-\lambda_{p} t}\left(\lambda_{p} t\right)^{r}}{r !}=t^{4 N} e^{-\lambda_{p} t} \sum_{r=1}^{\infty} \frac{\left(\lambda_{p} t\right)^{r}}{r^{4 N} r !} \\
& \leq \varepsilon^{-4 N} e^{-\lambda_{p} t} \sum_{r=1}^{\infty} \frac{\left(\lambda_{p} t\right)^{r+4 N}}{r^{4 N} r !} \\
& \leq \varepsilon^{-4 N} e^{-\lambda_{p} t}(1+4 N)^{4 N} \sum_{r=1}^{\infty} \frac{\left(\lambda_{p} t\right)^{r+4 N}}{(r+4 N) !} \\
& \leq \varepsilon^{-4 N}(1+4 N)^{4 N} .
\end{aligned}
$$

Substituting (20) and (21) into (19), we obtain (17). The theorem is proved.

\section{BIBLIOGRAPHY}

1. M. Thomas, A generalization of Poisson's binomial limit for use in ecology, Biometrica 36 (1949), 18-25. MR 11:528d

2. A. C. Gleeson and I. B. Douglas, Quadrat sampling and the estimation of Neyman type A and Thomas distributional parameters, Austral. J. Statist. 17 (1975), no. 2, 103-113.

3. A. V. Skorokhod, Stochastic Processes with independent Increments, "Nauka", Moscow, 1986; English transl., Kluwer, Dordrecht, 1991. MR 88b:60171 
O. G. KUKUSH AND YU. S. MISHURA

4. Yu. M. Kabanov, R. S. Liptzer, and A. N. Shiryaev, Martingale methods in the theory of point processes, Proceedings of the School-Seminar on the Theory of Random Processes, Part II, Vilnius, 1975, pp. 269-354. MR 55:4371

5. I. A. Ibragimov and R. Z. Khas'minskiŭ, Statistical Estimation: Asymptotic Theory, "Nauka", Moscow, 1979; English transl., Springer-Verlag, Berlin-Heidelberg-New York, 1981. MR 81h:62004

Department of Mathematical Analysis, Faculty for Mathematics and Mechanics, Kyiv National Taras Shevchenko University, Academician Glushkov Avenue 6, Kyiv-127 03127 , UKRAINE

E-mail address: Alexander_Kukush@univ.kiev.ua

Department of Mathematical Analysis, Faculty for Mathematics and Mechanics, Kyiv National Taras Shevchenko University, Academician Glushkov Avenue 6, Kyiv-127 03127, UKRAINE

E-mail address: myus@univ.kiev.ua

Received 7/MAY/2002

Translated by YU. MISHURA 